\title{
Tumor growth rate as a metric of progression, response, and prognosis in pancreatic and intestinal neuroendocrine tumors
}

Clarisse Dromain 1* (D), Marianne E. Pavel ${ }^{2}$, Philippe Ruszniewski ${ }^{3,4}$, Alison Langley ${ }^{5}$, Christine Massien ${ }^{5,6}$, Eric Baudin ${ }^{7}$, Martyn E. Caplin ${ }^{8}$ and on behalf of the CLARINET Study Group

\begin{abstract}
Background: Lanreotide depot/autogel antitumor activity in intestinal/pancreatic neuroendocrine tumors (NETs) was demonstrated in the phase-3 CLARINET study (NCT00353496), based on significantly prolonged progressionfree survival (PFS) versus placebo.

Methods: During CLARINET, patients with metastatic intestinal/pancreatic NETs received lanreotide depot/autogel $120 \mathrm{mg}$ or placebo every 4 weeks for 96 weeks. Imaging data (response evaluation criteria in solid tumors [RECIST] v1.0, centrally reviewed) were re-evaluated in this post hoc analysis of tumor growth rate (TGR) in NETs. TGR (\%/month) was calculated from two imaging scans during relevant periods: pre-treatment (TGRo); 12-24 weeks before randomization versus baseline; each treatment visit versus baseline $\left(T G R_{T x-0}\right)$; between consecutive treatment

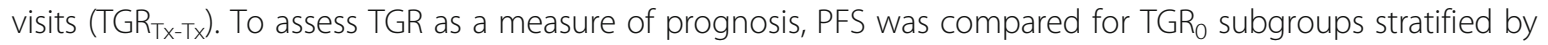
optimum TGRo cut-off; a multivariate analysis was conducted to identify prognostic factors for PFS.

Results: TGRo revealed tumors growing during pre-treatment (median [interquartile range] TGRo: lanreotide 2.1\%/ month $[0.2 ; 6.1]$; placebo $2.7 \%$ /month $[0.15 ; 6.8]$ ), contrary to RECIST status. TGR was significantly reduced by 12 weeks with lanreotide versus placebo (difference in least-square mean $\mathrm{TGR}_{0-12}$ of $-2.9[-5.1,-0.8], p=0.008$ ), a difference that was maintained at most subsequent visits. TGR $>4 \% /$ month had greater risk of progression/death than $\leq 4 \%$ /month (hazard ratio 4.1; [95\% Cl 2.5-6.5]; $p<0.001$ ); multivariate analysis revealed lanreotide treatment, progression at baseline, TGRo, hepatic tumor load, and primary tumor type were independently associated with PFS.

Conclusions: TGR provides valuable information on tumor activity and prognosis in patients with metastatic intestinal/pancreatic NETs, and identifies early lanreotide depot/autogel antitumor activity.
\end{abstract}

Trial registration: Retrospective registration, 18 July 2006; EudraCT: 2005-004904-35; ClinicalTrials.gov: NCT00353496.

Keywords: Lanreotide, Neuroendocrine tumor, Prognostic factor, RECIST, Tumor growth rate

\footnotetext{
* Correspondence: Clarisse.Dromain@chuv.ch

${ }^{1}$ Department of Diagnostic and Interventional Radiology, CHUV University

Hospital, Lausanne, Switzerland

Full list of author information is available at the end of the article
}

(C) The Author(s). 2019 Open Access This article is distributed under the terms of the Creative Commons Attribution 4.0 International License (http://creativecommons.org/licenses/by/4.0/), which permits unrestricted use, distribution, and reproduction in any medium, provided you give appropriate credit to the original author(s) and the source, provide a link to the Creative Commons license, and indicate if changes were made. The Creative Commons Public Domain Dedication waiver (http://creativecommons.org/publicdomain/zero/1.0/) applies to the data made available in this article, unless otherwise stated. 


\section{Background}

Neuroendocrine tumors (NETs) are slow-growing neoplasms that arise from diverse locations, including the pancreas and gastrointestinal (GI) tract. The prognosis of NETs is heterogeneous and tumor progression has been utilized in most phase-3 clinical trials to refine prognostic stratification. Current knowledge of well-differentiated NET growth kinetics and the relationship to treatment are limited; further understanding may improve the assessment of NET progression, prognosis and ultimately treatment.

Response evaluation criteria in solid tumors (RECIST) is used by oncologists and radiologists, and recognized by regulatory bodies as an assessment of tumor response to therapy, including NETs $[1,2]$. RECIST estimates change in tumor burden using the sum of the longest diameters (SLD) of target lesions over a course of treatment which, together with the appearance of new lesions, is transformed into a categorical variable (complete response $[\mathrm{CR}]$, partial response [PR], stable disease [SD], or progressive disease [PD]) $[1,2]$.

Although a valuable tool widely used in clinical trials, RECIST is a qualitative variable, overemphasizing tumor shrinkage as a successful response to treatment, and requires improved reproducibility [3, 4]. Limitations of RECIST extend to accurate assessment of NET disease status, which can be confounded by their slow growth kinetics [5]. As a result, it can be difficult for practitioners to decide how to adapt treatment. Therefore, a metric that provides a more sensitive measure of tumor growth would prove advantageous in slow-growing tumor assessment, including NETs.

An assessment providing dynamic and quantitative evaluation of tumor kinetics may offer a useful complement to RECIST. Tumor slope has been explored as a prognostic factor in studies on solid tumors [6-10], including well-differentiated NETs [9, 10]. Tumor growth rate (TGR), which is based on change in tumor volume, has been independently associated with progression-free survival (PFS) using data from phase-1 studies in solid tumors (including upper GI and pancreatic tumors) [11], and with PFS and overall survival (OS) using data from the phase-3 TARGET study (sorafenib compared with placebo) in metastatic renal cell carcinoma (mRCC) [12]. More recently, an association of TGR with PFS has been reported in a post hoc analysis from a phase-2 single-arm trial of lanreotide depot/autogel $120 \mathrm{mg}$ for non-functioning intestinal/pancreatic NETs in Japanese patients [13].

The CLARINET core study demonstrated the antitumor efficacy of lanreotide depot/autogel $120 \mathrm{mg} / 4$ weeks in patients with non-functioning intestinal/pancreatic NETs compared with placebo [14]. The CLARINET open-label extension confirmed long-term safety and efficacy [15]. Most participants in the core study had SD at baseline according to RECIST v1.0. In these post hoc analyses, core study tumor growth measurements were evaluated using TGR as a measure of tumor progression and response before and during treatment, and as a prognostic factor for PFS.

\section{Methods \\ Patients and study design}

The design and methods for the CLARINET study have been described previously [14]. In brief, patients had unresectable, locally advanced or metastatic, well/moderately differentiated, somatostatin receptor-positive NETs with Ki-67 up to $10 \%$. NETs were non-functioning except for gastrinomas controlled by proton-pump inhibitors for $\geq 4$ months. Tumors originated in the pancreas, midgut or hindgut, or were of unknown origin.

The CLARINET study was an international, randomized, double-blind, placebo-controlled phase-3 trial (EudraCT: 2005-004904-35; ClinicalTrials.gov: NCT00353496). Patients received lanreotide $120 \mathrm{mg}(n=101)$ or placebo $(n$ $=103$ ) once every 4 weeks, for 96 weeks or until PD (assessed centrally using RECIST v1.0) or death. The study was conducted in accordance with the Declaration of Helsinki, Good Clinical Practice Guidelines, and local regulatory requirements. Trial documentation was approved by institutional review boards at each study site, and all patients provided written informed consent. A full list of the ethics committees and their institutions is provided Appendix 1 of in the Additional file 1.

\section{Assessments and endpoints}

Study visits were scheduled during the screening period and at weeks 1 (baseline), 12, 24, 36, 48, 72, and 96 [14]. Baseline disease-progression status according to RECIST v1.0 was determined over a 12 - to 24-week screening period [14]. Computed tomography or magnetic resonance imaging of the chest, abdomen and pelvis was performed twice during screening. Changes in target-lesion sizes were assessed from the second imaging test (performed 12-24 weeks after the first scan), and randomization took place within the following 4 weeks. Single scans were obtained at post-baseline visits and reviewed centrally according to RECIST v1.0. If a patient was withdrawn from the study prematurely for reasons other than PD/death, further imaging was undertaken (unless already undertaken within the previous 4 weeks).

TGR was expressed as the percentage change in tumor volume over 1 month (\%/month): TGR $=100 \times(\exp (\mathrm{TG})-$ 1 ), where $\mathrm{TG}=3 \times \log (\mathrm{D} 2 / \mathrm{D} 1) /$ time (months) [11, 12]. Tumor size (D) was determined using the SLD of target lesions only (according to RECIST v1.0); non-target and new lesions were not considered. D1 and D2 represent tumor sizes at evaluation dates 1 and 2; and time $($ months $)=($ date $2-$ date $1+1) / 30.44$. 
The clinical utility of TGR was assessed: by evaluating pre-treatment TGR $\left(\mathrm{TGR}_{0}\right)$ using the two scans performed during screening; by calculating the change in TGR between pre-treatment and a visit during treatment $\left(\mathrm{TGR}_{\mathrm{Tx}-0}\right)$; and by calculating the TGR between consecutive visits during treatment $\left(\mathrm{TGR}_{\mathrm{Tx}-\mathrm{Tx}}\right)$. To investigate the value of $\mathrm{TGR}_{0}$ as a prognostic factor for tumor progression (according to RECIST v1.0), the optimum $\mathrm{TGR}_{0}$ cut-off value (\%/month) was determined, and PFS was compared for $\mathrm{TGR}_{0}$ subgroups stratified by the optimum $\mathrm{TGR}_{0}$ cut-off. Exploratory analyses were also conducted to identify potential prognostic factors for PFS (according to RECIST v1.0). Analyses were carried out to determine whether Ki-67 correlated with $\mathrm{TGR}_{0}$ in all patients with a tumor biopsy; and in a subgroup of patients with a tumor biopsy taken within 1 year of starting treatment.

\section{Statistical methods}

A priori summary statistics were prepared for baseline characteristics based on the intention-to-treat (ITT) population (all randomized patients) [14]. All other analyses were post hoc and based on the ITT population, or a specified subset thereof. Spearman's rank correlation was used to test for an association between Ki-67 at screening and $\mathrm{TGR}_{0}$. TGRs during the study were analyzed using mixed-model regression analyses with repeated measures; least square (LS) means and 95\% confidence intervals (CIs) were calculated by visit for each treatment.

To explore TGR as a prognostic factor, a receiver operating characteristic (ROC) analysis was conducted to determine the optimum $\mathrm{TGR}_{0}$ cut-off value associated with risk of PD (according to RECIST v1.0) or death. PFS was estimated using the Kaplan-Meier method and compared between lanreotide and placebo according to subgroups defined by the $\mathrm{TGR}_{0}$ cut-off value using the log-rank test. Comparisons were also made between the $\mathrm{TGR}_{0}$ subgroups within each treatment group. In an additional analysis, PFS was estimated between lanreotide and placebo for the subgroups $\mathrm{TGR}_{0}>4 \% /$ month and $\leq 10 \% /$ month; and $\mathrm{TGR}_{0}>10 \% /$ month. To explore the prognostic value of absolute TGR at 12 weeks ( $\mathrm{TGR}_{0-12}$ ), an association between $\mathrm{TGR}_{0-12}$ and PFS was assessed for lanreotide and placebo groups. Hazard ratios (HRs) were estimated from Cox proportional hazard models. Patients with PD due to non-target or new lesions were excluded from these subgroup analyses. The prognostic value of $\mathrm{TGR}_{0}$ was further explored using a Cox proportional hazards model to identify potentially important covariates (Wald Chi-square $p<0.10$, Additional file 1: Table S1). These covariates were analyzed via a multivariate Cox proportional hazard model of PFS; only covariates potentially important in the presence of other terms $(p<0.10)$ remained in the final model. All statistical analyses were carried out using SAS statistical software (SAS V9.4).

\section{Results}

\section{Patients}

The patient population has been described previously [14]. Briefly, 204 patients were randomized (ITT population) to receive lanreotide $(n=101)$ or placebo $(n$ $=103)$. Most patients had received no previous treatment $(84 \%, 172 / 204)$ and had SD over the 12 - to 24-week screening period before study entry (96\%, 195/ 204), according to RECIST v1.0 [14]. Mean (standard deviation) time between diagnosis and enrollment was 33.5 (43.7) months. All patients had grade 1 or low-grade 2 tumors (Ki-67<10\%). Overall, 33\% (67/204) of patients had hepatic tumor loads over 25\%; $45 \%$ (91/204) had primary tumors of the pancreas and $36 \%$ (73/204) of the midgut. In total, 200 patients with evaluable data and available SLD measurements were included in these post hoc analyses. Patients were excluded if they had: missing SLD; a change in TGR that could not be measured; or non-target or new lesions (Additional file 1: Figure S1).

\section{Use of TGR to measure tumor progression and response before and during treatment}

The distributions of patient $\mathrm{TGR}_{0}$ during the pre-treatment period were similar for both treatment groups (post hoc analyses; ITT population; Fig. 1a). Overall, patients receiving lanreotide had a median (interquartile range) $\mathrm{TGR}_{0}$ of $2.1 \% /$ month (0.2-6.1), while those receiving placebo had $2.7 \% /$ month $(0.15-6.8)$. The majority of patients, including those with SD, had increases in SLD measurements during the pre-treatment period (Fig. 1b). We observed no correlation between $\mathrm{Ki}-67$ at screening and $\mathrm{TGR}_{0}$ in analyses that included either all patients with a tumor biopsy, or a subgroup of patients with a tumor biopsy taken within 1 year of starting treatment (Additional file 1: Figure S2).

When using TGR to measure proliferative activity during treatment, variation between $\mathrm{TGR}_{0}$ and each study visit $\left(\mathrm{TGR}_{\mathrm{Tx}-0}\right)$ according to RECIST v1.0 status revealed that a large proportion of patients had reductions in TGR at post-treatment visits relative to $\mathrm{TGR}_{0}$ (Additional file 1 : Figure S3), with greater reductions in lanreotide compared with the placebo group. These findings were despite most patients being classified as SD with RECIST v1.0, regardless of treatment. A disparity between median $\mathrm{TGR}_{0}$ and LS mean $\mathrm{TGR}_{0}$ in the lanreotide group was due to high $\mathrm{TGR}_{0}$ in two patients. Calculation of TGRs between consecutive visits during treatment $\left(\mathrm{TGR}_{\mathrm{Tx}-\mathrm{Tx}}\right)$ revealed a reduction in TGR as early as week 12 of lanreotide treatment $\left(\mathrm{TGR}_{0-12}\right)$, resulting in a statistically significant difference between treatment groups that was maintained throughout most of the treatment period (Fig. 2; Additional file 1: Table S2). Individual TGRs $\left(\mathrm{TGR}_{\mathrm{Tx}-\mathrm{Tx}}\right)$ for patients with $\mathrm{PD}$ after the start of treatment revealed that, in many cases, PD was a result of accumulated tumor growth over time, rather than a sudden increase in TGR (Additional file 1: Figure S4). 

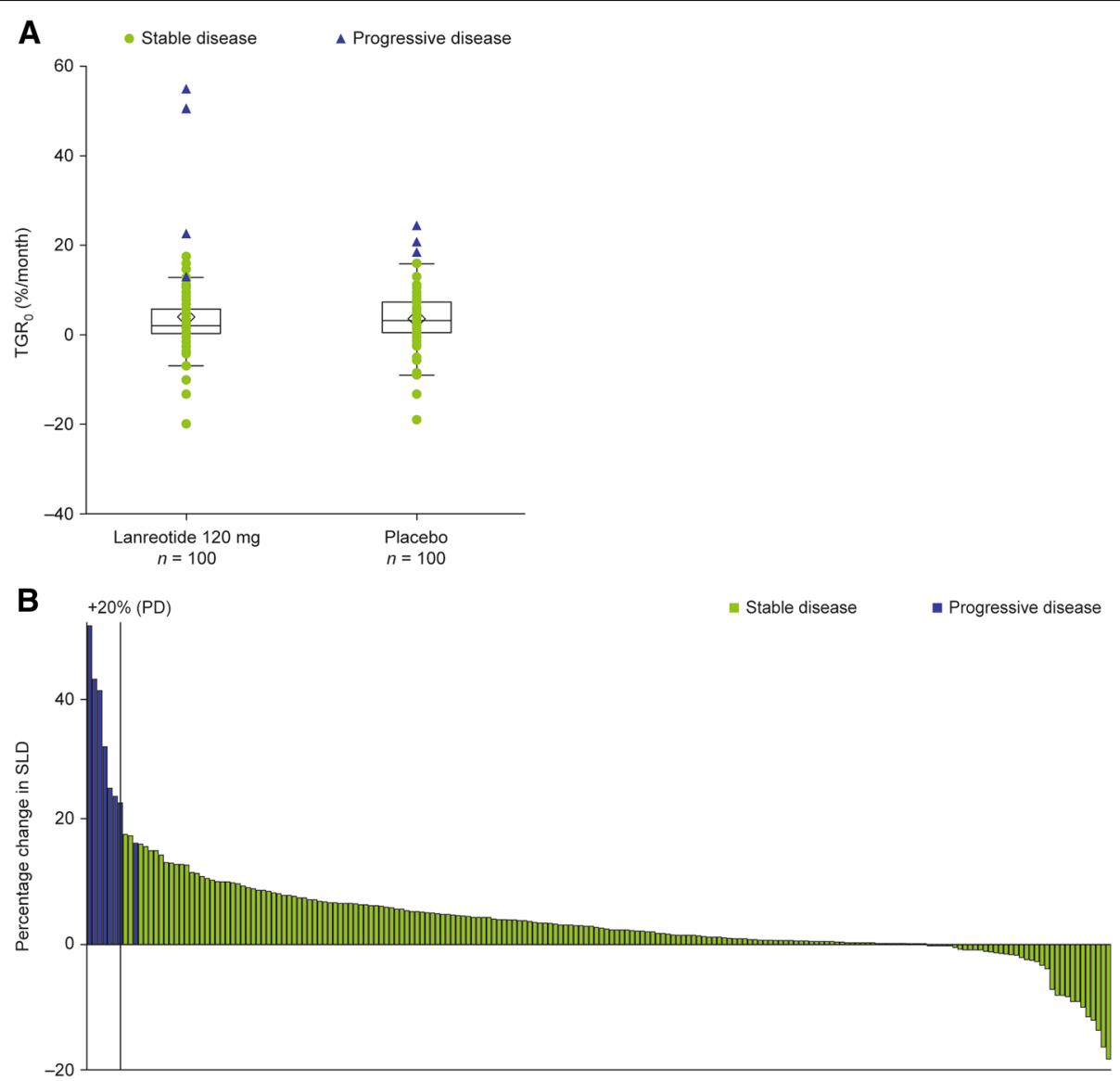

Fig. 1 Distribution of (a) TGRo and (b) percentage change in SLD among patients during the pre-treatment period. a Distribution of TGRO among patients during the pre-treatment period according to RECIST v1.0 classification. Individual data points represent the TGRO in individual patients (ITT population), according to their classification by RECIST v1.0. Boxes represent median and interquartile range (Q1-Q3), whiskers represent the minimum observation still within 1.5 IQR of lower quartile (Q1), and the maximum observation still within 1.5 IQR of the upper quartile (Q3). Median (IQR) TGRO: 2.1\%/month $(0.2 ; 6.1)$ (lanreotide $120 \mathrm{mg}) ; 2.7 \%$ /month $(0.15 ; 6.8)$ (placebo). b Percentage changes in SLD during the pretreatment period among individual patients (ITT population). Data are sorted from high percentage change in SLD to low percentage change in SLD over the pre-treatment period. A patient with a change in SLD less than 20\% had PD due to the presence of new lesions. Lanreotide 120 $m g, n=100 ;$ placebo, $n=100$. ITT, intention-to-treat; IQR; interquartile range; PD, progressive disease; RECIST, response evaluation criteria in solid tumors; SLD, sum of longest diameters; TGRO, pre-treatment tumor growth rate

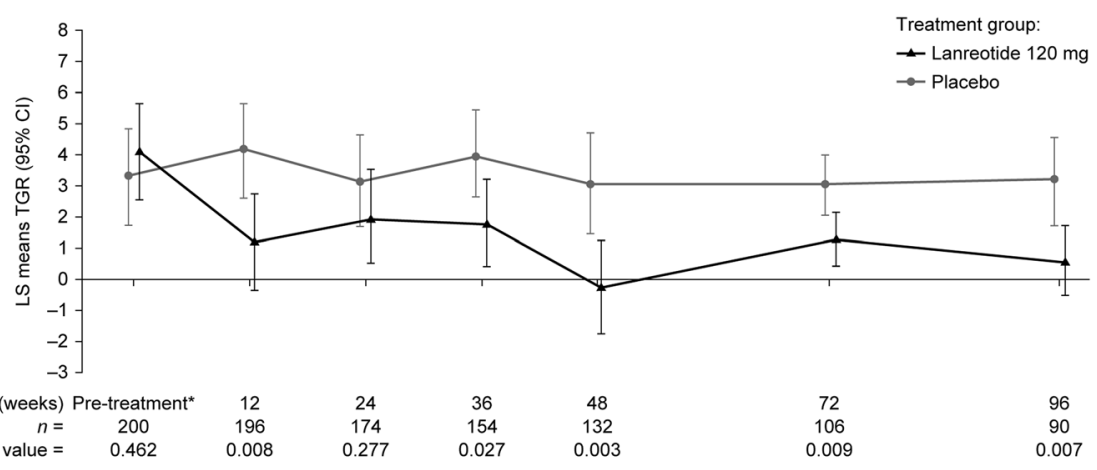

Fig. 2 Estimated $T G R_{0}$ and $T G R_{T x-T x}$. ${ }^{*}$ Pre-treatment $T G R\left(T G R_{0}\right)$ is calculated from the second imaging test during the screening period (performed 12-24 weeks after the first screening period scan). LS means and $P$-values are derived from a mixed model with repeated measures. $\mathrm{Cl}$, confidence interval; $L S$, least squares; TGR, tumor growth rate; $T G R_{0}$, pre-treatment $T G R, T G R_{T x-T x}, T G R$ between consecutive study visits during treatment 


\section{Use of $\mathrm{TGR}_{0}$ as a prognostic factor}

Of the $\mathrm{TGR}_{0}$ thresholds assessed by ROC analysis, a cut-off $\mathrm{TGR}_{0}$ of $4 \% /$ month during the pre-treatment period had optimal association with the risk of PD (according to RECIST v1.0 status) or death independently of treatment group (Additional file 1: Figure S5). When comparing PFS between $\mathrm{TGR}_{0}$ subgroups, $\mathrm{TGR}_{0}>4 \% /$ month was associated with a four-fold greater risk of $\mathrm{PD} /$ death than $\mathrm{TGR}_{0} \leq$ $4 \%$ month in the overall population (HR 4.1 [95\% CI [2.56.5]; $p<0.001, n=187$ ), and within each treatment group (Additional file 1: Figure S6). When considering PFS within $\mathrm{TGR}_{0}$ subgroups between treatment groups, lanreotide was significantly more effective than placebo at reducing the risk of $\mathrm{PD} /$ death, by 73 and $63 \%$ in those with $\mathrm{TGR}_{0} \leq 4 \%$ / month and $>4 \% /$ month, respectively (Fig. 3 ). In a separate analysis, lanreotide reduced the risk of $\mathrm{PD} /$ death by $73 \%$ compared with placebo in a subgroup of patients with $\mathrm{TGR}_{0}>4 \% /$ month and $\leq 10 \% /$ month (Additional file 1 : Figure S7). There was a trend towards increased PFS with lanreotide compared with placebo in patients with $\mathrm{TGR}_{0}>$ $10 \% /$ month, however this did not reach statistical significance. Thirteen patients considered to show disease progression based only on non-target or new lesions were excluded from these analyses (lanreotide, $n=6$; placebo, $n=7)$. The prognostic value of early on-treatment TGR $\left(\mathrm{TGR}_{0-12}\right)$ was also explored. Analyses within each treatment group indicated that a higher $\mathrm{TGR}_{0-12}$ was associated with worse PFS in both lanreotide (HR for a $10 \% /$ month increase in $\mathrm{TGR}_{0-12}$ : 8.0 [95\% CI [3.0; 21.3], $p<0.0001, N=90)$ and placebo groups (HR for a $10 \% /$ month increase in $\mathrm{TGR}_{0-12}: 8.8$ [95\% CI $[4.4 ; 17.6], p<0.0001, N=93)$.

An exploratory analysis of 15 potential prognostic factors for PFS identified seven potentially important covariates $(p<0.10$; Additional file 1: Table S1). A separate analysis, restricted to the subgroup of patients with tumor biopsies taken within 1 year of treatment start, confirmed that neither Ki-67 ( $p=0.5917 ; N=126)$ nor tumor grade ( $p=0.5294 ; N=125)$ were potentially important prognostic factors for PFS in this subgroup of patients. When the seven potentially important covariates were included into a multivariate model, chromogranin A (CgA) levels at baseline, body mass index (BMI), sex and tumor grade were no longer significant at $p=0.10$ in the presence of the other terms and were, therefore, excluded from the final multivariate model. The final model showed $\mathrm{TGR}_{0}>4 \%$, hepatic tumor load $(>25 \%-\leq 50$ and $>50 \%$ ), progression at baseline, and a pancreatic primary tumor were independent prognostic factors for worse PFS (according to RECIST v1.0), while prior therapy was not prognostic for PFS in the presence of other terms (Fig. 4). After adjustment for these prognostic factors, lanreotide reduced the risk of progression or death by $69 \%$ versus placebo. Risk of $\mathrm{PD} /$ death was more than three-fold higher in patients with $\mathrm{TGR}_{0}>4 \% /$ month.

\section{Discussion}

These post hoc analyses were conducted to evaluate the clinical utility of TGR as a measure of tumor progression, response and prognosis in patients with intestinal/ pancreatic NETs receiving lanreotide or placebo, using data from the CLARINET study [14]. Analyses of the use of TGR to assess tumor progression before treatment $\left(\mathrm{TGR}_{0}\right)$ revealed a large proportion of patients' tumors were actively growing during the pre-treatment period, despite classification as SD according to RECIST v1.0. There was no correlation between Ki-67 at screening and $\mathrm{TGR}_{0}$, despite an attempt to stratify the data by only including patients with biopsies taken within 1 year of treatment start. Analyses of the use of TGR to assess proliferative activity during treatment $\left(\mathrm{TGR}_{\mathrm{Tx}-0}\right)$ showed a large proportion of patients had reductions in $\mathrm{TGR}_{\mathrm{Tx}-}$ 0 , which tended to be greater with lanreotide compared
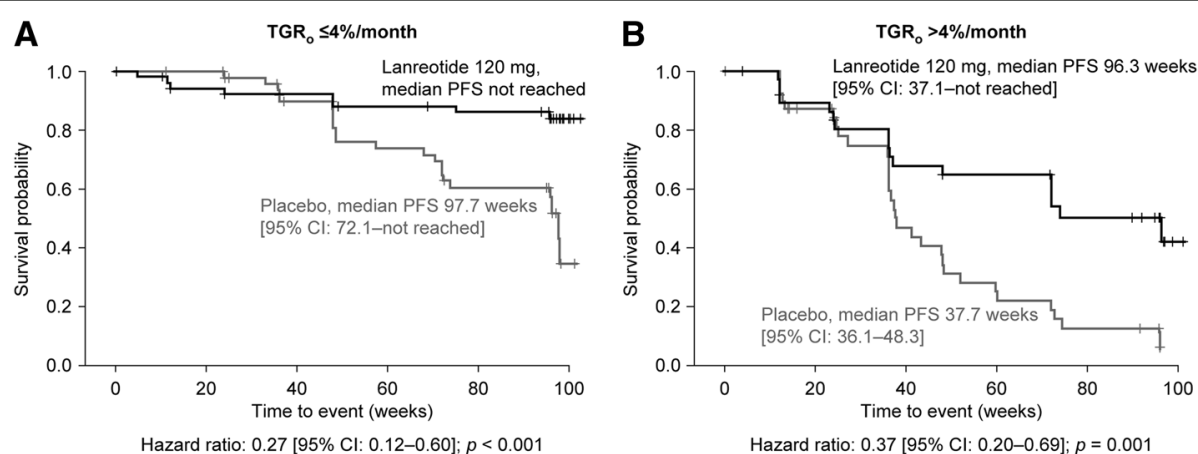

Fig. 3 PFS in patients with (a) $T G R_{0} \leq 4 \% / m o n t h$ and (b) $T G R_{0}>4 \% / m o n t h$. Analysis of PFS considers centrally assessed disease progressions (using RECIST v1.0 criteria) and any deaths reported during the study as events. A total of 14 patients in progression purely due to non-target or new lesions were excluded. TGR $\leq 4 \%$, lanreotide $n=56$; placebo $n=53 ; \mathrm{TGR}_{0}>4 \%$, lanreotide $n=38$; placebo $n=40$. HRs are derived from a Cox proportional hazards model. P-values are derived from the log-rank test. Cl, confidence interval; HR, hazard ratio; PFS, progression-free survival; TGR, tumor growth rate; $T G R_{0}$, pre-treatment TGR 


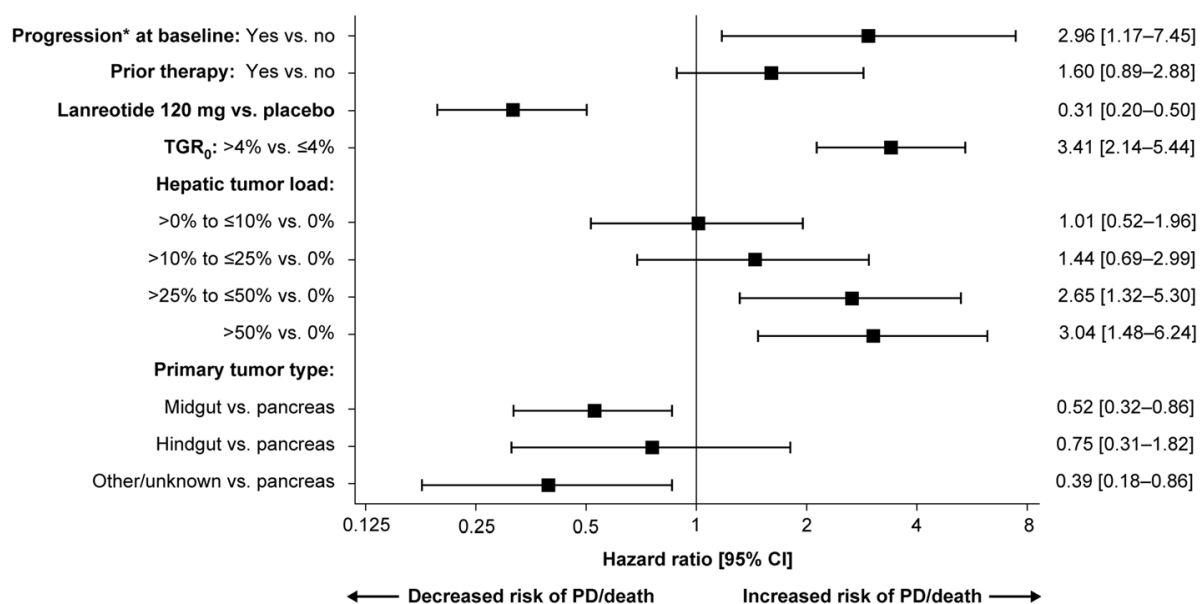

Fig. 4 Potential prognostic factors for PFS (final multivariate model). *According to RECIST v1.0. Hazard ratios [95\% CI] for centrally assessed PD or death were generated from a post hoc multivariate Cox proportional hazards model. Four patients with missing TGRo were excluded from the model. There were limited patients with progression at baseline $(n=8)$ and primary tumor type: hindgut $n=14$. Cl, confidence interval; PD, progressive disease; PFS, progression-free survival; RECIST, response evaluation criteria in solid tumors; TGR, pre-treatment tumor growth rate

with placebo, while most patients were still classified as SD according to RECIST v1.0. $\mathrm{TGR}_{\mathrm{Tx}-\mathrm{Tx}}$ demonstrated the antitumor efficacy of lanreotide compared with placebo, as early as 12 weeks into treatment, by reduction and subsequent stabilization. Analyses of the use of $\mathrm{TGR}_{0}$ as a prognostic factor showed that by using a $\mathrm{TGR}_{0}$ cut-off of $4 \% /$ month (found to have optimal association with risk of $\mathrm{PD} /$ death by ROC analysis), patients with $\mathrm{TGR}_{0}>4 \% /$ month had a four-fold higher risk of $\mathrm{PD} /$ death compared with $\leq 4 \% /$ month, in the overall population and within both treatment groups. $\mathrm{TGR}_{0-12}$ was also found to be prognostic for PFS in both treatment groups. A multivariate analysis identified five factors independently associated with PFS: lanreotide treatment, progression at baseline, $\mathrm{TGR}_{0}$, hepatic tumor load ( $>25 \%-\leq 50$ and $>50 \%$ ), and primary tumor type. Ki-67 at baseline was not identified as a potentially important prognostic factor for PFS, prior therapy (yes) was not prognostic in the presence of other terms and, notably, CgA at baseline, and tumor grade were excluded from the final model.

The findings from the present analyses accord with other similar analyses. The advantage of TGR as a rapid measure of tumor response has been reported previously; TGR identified antitumor efficacy during treatment at the first tumor evaluation in phase- 1 and -3 studies of mRCC $[11,12,16]$. The utility of TGR in non-functioning intestinal/pancreatic NETs was first described in a recent single-arm phase- 2 trial of 32 Japanese patients [13]. A numerical reduction in TGR was identified within 12 weeks of initiating lanreotide treatment, despite $65 \%$ of patients being classified as PD at baseline (RECIST v1.1), and was sustained from pre-treatment to last-value [13]. Our analyses expand upon this study in four ways: firstly, they reveal that TGR can detect early differences between lanreotide and placebo groups, despite the slow growth kinetics of NETs; secondly, they demonstrate sustained lanreotide antitumor activity compared with placebo using the large dataset from CLARINET; thirdly they provide additional evidence that although lanreotide reduced TGR versus placebo overall, tumor growth was ongoing in many patients, including those classified as having SD; and finally, in many cases a PD event was the result of ongoing tumor growth, rather than the result of a sudden increase in TGR. Our findings regarding the utility of TGR during treatment $\left(\mathrm{TGR}_{\mathrm{Tx}}\right)$ for monitoring tumor response and the durability of drug effect also resonate with other analyses from phase- 2 and -3 trials in other tumor types $[6,12,17,18]$. TGR 0 measurements were suggestive of actively growing tumors and, as seen with other tumor types, $\mathrm{TGR}_{0}$ did not reflect RECIST status during pre-treatment and early treatment evaluation $[11,12,19]$. Others have highlighted the inadequacies of RECIST as a measure of tumor response because it involves condensing information into four categories that are defined before treatment, regardless of growth kinetics. Additionally, RECIST may not always be relevant for slow-growing tumors or treatments that stabilize growth, and any observed pseudoprogression, even if uncommon in NETs, may be misrepresented as PD by RECIST $[3,19,20]$. Our findings that a $\mathrm{TGR}_{0}$ cut-off of $4 \% /$ month and $\mathrm{TGR}_{0-12}$ are prognostic for PFS are consistent with others who identified an association between growth kinetics and clinical outcomes. In a large retrospective analysis of 20 phase- $1 \mathrm{mRCC}$ trials, a $9 \%$ decrease in progression hazard was observed with every 10\% decrease in TGR (reference compared with experimental period) [11], and TGR was associated with PFS regardless of treatment (sorafenib or 
placebo) in a retrospective analysis from the phase- 3 TARGET trial in mRCC [12]. A new response metric for glioblastoma incorporating a linear model of radial tumor expansion computed at first post-radiation scan was prognostic for PFS, and agreed with more complex anatomic and spherical equivalents [21, 22]. In addition, response outcomes utilizing growth dynamics have also been previously shown to be prognostic for OS in renal and prostate cancer $[12,17,18,21-23]$. The $\mathrm{TGR}_{0}$ cut-off of $4 \% /$ month could, therefore, be of value within routine clinical practice by providing a prognosis for PFS before treatment start, thus allowing clinicians to make earlier decisions regarding future treatment. $\mathrm{TGR}_{\mathrm{Tx}-0}$ would also be potentially useful in the clinic to identify patients who are or are not benefitting early in the course of their treatment (e.g. $\left.\mathrm{TGR}_{12-0}\right)$. $\mathrm{TGR}_{0}(\leq />4 \% /$ month), if confirmed to be prognostic for other therapies, and $\mathrm{TGR}_{\mathrm{Tx}-0}$ would be of particular importance when planning and monitoring more toxic treatments, such as peptide receptor radionuclide therapy and chemotherapy, ensuring these are continued only for as long as is necessary.

The lack of correlation between $\mathrm{Ki}-67$ and $\mathrm{TGR}_{0}$ in this analysis was surprising. However, known difficulties in assessment of the Ki-67 index (intra- and intertumoral staining heterogeneity and counting methods, for example) may, in part, account for this [24, 25]. Ki-67 was not identified as a potentially important prognostic factor for PFS from the exploratory multivariate analysis, despite the known importance of Ki-67 as a prognostic marker in NETs [26]. Nevertheless, our findings accord with a previous exploratory analysis of prognostic factors using data from the CLARINET study [27]. Despite being of potential interest in the univariate setting, CgA levels at baseline, tumor grade, BMI, and sex were excluded from the final multivariate model presented here. Reassuringly, this resonates with previous analyses of prognostic factors for PFS [13, 27], suggesting CgA levels and tumor grade are less robust prognostic factors than hepatic tumor load and $\mathrm{TGR}_{0}$ in patients with NETs.

This study was not without limitations. There are inherent limitations in post hoc analyses that potentially limit their interpretation. Any confounding due to anisotropy was not accounted for, as target lesions were assumed to be spherical, although this does tend to be the case for liver metastases. Target lesions followed for TGR may be slow growing, and all lesions within a patient were assumed to be similar; therefore, TGR for an individual may not be representative of their overall tumor targets. Inaccuracies in TGR may be introduced by errors in SLD measurements and, as tumor growth was assumed to be exponential, deviations from this growth pattern; in addition, for $\mathrm{TGR}_{\mathrm{Tx}-0}$, lesions used for the tumor assessments during the screening period did not have to be the same lesions as those assessed during the treatment period. In concordance with the SLD calculation used in RECIST v1.0, non-target and new lesions were not considered in TGR calculations. The Ki-67 and $\mathrm{TGR}_{0}$ correlation analysis and exploratory analysis of the prognostic value of Ki-67 were limited due to a number of reasons. Firstly, many tumor biopsies were collected several years before the start of treatment, although additional analyses (restricted to the subgroup in whom biopsies were taken in the year before treatment initiation) provided similar results to the overall population. Secondly, Ki-67 data were either unreliably quantified or missing for 41 patients who were enrolled into the CLARINET study based on mitotic index. Thirdly, a number of patients had Ki-67 values recorded as $<1 \%(N=15)$ or $<2 \%(N=29)$, which restricted the way Ki-67 data could be handled; and few patients $(N=17)$ with Ki-67 values $>5 \%$ were included. The ROC area-under-curve implied that $\mathrm{TGR}_{0} 4 \% /$ month cut-off was not strongly deterministic of PFS. Further validation will be required to determine whether this cut-off is relevant in other study populations. Thirteen patients with progression based on non-target or new lesions were excluded from the prognostic value analysis of the $\mathrm{TGR}_{0} 4 \% /$ month cut-off. However, as a similar number of patients were excluded in each treatment group, it is unlikely to have affected the results.

These limitations notwithstanding, our findings suggest TGR has potential clinical utility as a novel metric for proliferative activity, particularly in future studies of somatostatin analogs or novel targeted therapies in NETs in which subtle changes in tumor growth are expected but may not be identified using RECIST. Further advantages of TGR utility include its potential in individualizing patient treatment, with the possibility that therapy can be adjusted based on a more precise analysis of tumor kinetics.

\section{Conclusion}

Overall, these post hoc analyses of TGR to assess activity before and during treatment revealed that $\mathrm{TGR}_{0}$ and $\mathrm{TGR}_{\mathrm{Tx}}$ provided valuable information on tumor kinetics. Evaluation of $\mathrm{TGR}_{0}$ as a prognostic factor revealed $\mathrm{TGR}_{0}$ was prognostic for PFS, irrespective of treatment. Furthermore, lanreotide was more effective than placebo in delaying $\mathrm{PD} /$ death, irrespective of the $\mathrm{TGR}_{0} 4 \% /$ month cut-off. Future perspectives include the development of TGR-based categorical definitions of disease status, and further validation of TGR as a predefined endpoint in prospective studies.

\section{Additional file}

Additional file 1: Table S1. Categories tested in the exploratory multivariate analyses of potential prognostic factors. Table S2. TGRO and 
TGRTx-Tx for patients receiving lanreotide or placebo (\%/month). Figure S1. Patient disposition in the CLARINET TGR analysis. Figure S2. Correlation between TGRO and Ki-67 at screening in (a) all patients and (b) in a subgroup of patients with a tumor biopsy taken within 1 year of the start of treatment (ITT population). Figure S3. Variation in TGR and tumor response evaluation by RECIST v1.0 between pre-treatment and each treatment visit (TGRTX-0) at (a) Week 12; (b) Week 24; (c) Week 36; (d) Week 48; (e) Week 72; and (f) Week 96. Figure S4. Individual TGRs (TGRTX-Tx) for patients with PD after the start of treatment within (a) lanreotide and (b) placebo treatment groups. Figure S5. Determination of the optimum TGRO cut-off value. Figure S6. PFS between TGRO subgroups ( $\leq 4 \% /$ month and $>4 \% /$ month) within (a) lanreotide and (b) placebo treatment groups. Figure S7. PFS between lanreotide and placebo groups within TGRO subgroups (a) $>4 \% /$ month and $\leq 10 \% /$ month and (b) $>10 \% /$ month. Appendix 1: List of Ethics Committees and/or Institutional Review Boards. (DOCX 1446 kb)

\section{Abbreviations}

BMl: Body mass index; CgA: Chromogranin A; Cl: Confidence interval; CR: Complete response; GI: Gastrointestinal; HR: Hazard ratio; ITT: Intentionto-treat; LS: Least square; mRCC: Metastatic renal cell carcinoma; NET: Neuroendocrine tumor; OS: Overall survival; PD: Progressive disease; PFS: Progression-free survival; PR: Partial response; RECIST: Response evaluation criteria in solid tumors; ROC: Receiver operating characteristic; SD: Stable disease; SLD: Sum of the longest diameters; TGR: Tumor growth rate

\section{Acknowledgments}

The authors thank all patients involved in the study, as well as their caregivers, care team, investigators and research staff in participating institutions. The authors thank Tom Vizard, PhD and Germanicus HansaWilkinson, MSc, of Watermeadow Medical, an Ashfield company, for providing medical writing and editorial support, which was funded by Ipsen in accordance with Good Publication Practice guidelines.

CLARINET Study Group

Austria M. Raderer; Belgium I. Borbath, D. Ysebaert; Czech Republic E. Sedláčková, P. Vítek; Denmark H. Grønbæk; France A. Adenis, L. Buscail, G. Cadiot, S. Dominguez, M. Ducreux, C. Lombard-Bohas, E. Mitry, P. Ruszniewski, J.F. Seitz; Germany N. Begum, I. Harsch, M. Pavel, C. Schöfl, M. Weber, B. Wiedenmann; India M. Mallath, P. Patil, K. Sambasivaiah, R. Saxena; Italy E. Bajetta, A. Buonadonna, R. Buzzoni, R. Cannizzaro, A. Colao, C. De Angelis, P. Tomassetti; Poland J. Ćwikła, B. Kos-Kudła; Slovakia T. Salek; Spain J. Capdevila, G. Soler, J.M. Tabernero; Sweden H. Ahlman, M. Kjellman; UK G. Aithal, A. Anthoney, M. Caplin, A. Grossman, J. Newell-Price, J. Ramage, N. Reed, A. Rees, W. Steward, L. Wall; USA M. Choti, A.T. Phan, E.M. Wolin.

\section{Funding}

Ipsen sponsored the original study, and ordered and funded the post-hoc analyses reported here. Ipsen funded the medical writing support and reviewed the manuscript draft. Two of the authors were employed by Ipsen at the time of the analysis.

\section{Availability of data and materials}

Where patient data can be anonymized, Ipsen will share all individual participant data that underlie the results reported in this article with qualified researchers who provide a valid research question. Study documents, such as the study protocol and clinical study report, are not always available. Proposals should be submitted to DataSharing@lpsen.com and will be assessed by a scientific review board. Data are available beginning 6 months and ending 5 years after publication; after this time, only raw data may be available.

\section{Authors' contributions}

CD contributed to the conceptualization, methodology, supervision, validation, visualization, writing - original draft, writing - review and editing for this manuscript. MEP contributed to the data curation, investigation, methodology, writing - original draft, writing - review and editing for this manuscript. PR contributed to the conceptualization, formal analysis, methodology, writing - original draft, writing - review and editing for this manuscript. AL contributed to the data curation, methodology, writing original draft, writing - review and editing for this manuscript. CM contributed to the conceptualization, methodology, supervision, validation, writing - original draft, writing - review and editing for this manuscript. EB contributed to the conceptualization, validation, writing - original draft, writing - review and editing for this manuscript. MEC contributed to the conceptualization, writing - original draft, writing - review and editing for this manuscript. All authors read and approved the final manuscript.

\section{Ethics approval and consent to participate}

Trial documentation was approved by institutional review boards at each study site, and all patients provided written informed consent. A full list of the ethics committees and their institutions is provided in Appendix 1 of the Additional file 1.

\section{Consent for publication}

Not applicable

\section{Competing interests}

CD: Consultant/Advisor: Ipsen; MEP: Consultant/Advisor: Ipsen, Lexicon, Novartis, Pfizer; Research Support: Ipsen, Novartis; PR: Consultant/Advisor: Ipsen; Speakers bureau: Ipsen, Novartis; Research Support: Ipsen, Novartis; Employee: Ipsen (Spouse); AL: Employee: Ipsen; CM: Employee: Ipsen (at the time of work); EB: Received remuneration for services (advisory board or board of directors; corporate-sponsored research; consulting fee; research investigator; speaker) from Ipsen, Novartis, Pfizer, and Advanced Accelerator Applications; MEC: Received honoraria, consultancy and speakers' bureau fees from Ipsen, Advanced Accelerator Applications, and Novartis.

\section{Publisher's Note}

Springer Nature remains neutral with regard to jurisdictional claims in published maps and institutional affiliations.

\section{Author details}

${ }^{1}$ Department of Diagnostic and Interventional Radiology, CHUV University Hospital, Lausanne, Switzerland. ²Department of Medicine 1, Division of Endocrinology and Diabetology, Friedrich-Alexander Universität Erlangen-Nürnberg, Universitätsklinikum Erlangen, Erlangen, Germany. ${ }^{3}$ Division of Gastroenterology and Pancreatology, Beaujon Hospital, Clichy, France. ${ }^{4}$ Faculty of Medicine, Paris Diderot University, Paris, France. ${ }^{5}$ Ipsen, Boulogne-Billancourt, France. ${ }^{6}$ APHP, Hypertension unit, Georges Pompidou European Hospital, F-75015 Paris, France. ${ }^{7}$ Endocrine Tumour and Nuclear Medicine Unit, Gustave-Roussy Cancer Campus, Villejuif, France.

${ }^{8}$ Neuroendocrine Tumour Unit, Department of Gastroenterology, Royal Free Hospital, London, UK.

Received: 17 August 2018 Accepted: 27 December 2018 Published online: 14 January 2019

\section{References}

1. Therasse P, Arbuck SG, Eisenhauer EA, Wanders J, Kaplan RS, Rubinstein L, Verweij J, Van Glabbeke M, van Oosterom AT, Christian MC, et al. New guidelines to evaluate the response to treatment in solid tumors. European Organization for Research and Treatment of Cancer, National Cancer Institute of the United States, National Cancer Institute of Canada. J Natl Cancer Inst. 2000;92(3):205-16.

2. Eisenhauer EA, Therasse P, Bogaerts J, Schwartz LH, Sargent D, Ford R, Dancey J, Arbuck S, Gwyther S, Mooney M, et al. New response evaluation criteria in solid tumours: revised RECIST guideline (version 1.1). Eur J Cancer. 2009;45(2):228-47.

3. Sharma MR, Maitland ML, Ratain MJ. RECIST: no longer the sharpest tool in the oncology clinical trials toolbox---point. Cancer Res. 2012;72(20):5145-9 discussion 5150.

4. Abramson RG, McGhee CR, Lakomkin N, Arteaga CL. Pitfalls in RECIST data extraction for clinical trials: Beyond the Basics. Acad Radiol. 2015;22(6):779-86.

5. Sundin A, Rockall A. Therapeutic monitoring of gastroenteropancreatic neuroendocrine tumors: the challenges ahead. Neuroendocrinology. 2012; 96(4):261-71.

6. Kappeler C, Reichardt P, Kang Y-K, Blay J-Y, Joensuu H, Schaefer K, Chung J, Wagner A, Casali PG, Demetri G. O-016 exploratory analysis of tumor growth rate in patients with advanced gastrointestinal stromal tumors (GIST) treated with regorafenib in the GRID phase 3 trial. Ann Oncol. 2015; 26(suppl 4):iv114. 
7. Kappeler C, Healy DP, Baumer C, Meinhardt G, Elisei R, Schlumberger M, Brose MS. Analysis of tumor growth rate for radioiodine (RAl)-refractory differentiated thyroid cancer patients receiving placebo and/or sorafenib in the phase III DECISION study. J Clin Oncol. 2015;33(suppl):abst 6015.

8. Madeira I, Terris B, Voss M, Denys A, Sauvanet A, Flejou JF, Vilgrain V, Belghiti J, Bernades P, Ruszniewski P. Prognostic factors in patients with endocrine tumours of the duodenopancreatic area. Gut. 1998;43(3):422-7.

9. Palazzo M, Lombard-Bohas C, Cadiot G, Matysiak-Budnik T, Rebours V, Vullierme MP, Couvelard A, Hentic O, Ruszniewski P. Ki67 proliferation index, hepatic tumor load, and pretreatment tumor growth predict the antitumoral efficacy of lanreotide in patients with malignant digestive neuroendocrine tumors. Eur J Gastroenterol Hepatol. 2013;25(2):232-8.

10. Durante C, Boukheris H, Dromain C, Duvillard P, Leboulleux S, Elias D, de Baere T, Malka D, Lumbroso J, Guigay J, et al. Prognostic factors influencing survival from metastatic (stage IV) gastroenteropancreatic well-differentiated endocrine carcinoma. Endocr Relat Cancer. 2009;16(2):585-97.

11. Ferte C, Fernandez M, Hollebecque A, Koscielny S, Levy A, Massard C, Balheda R, Bot B, Gomez-Roca C, Dromain C, et al. Tumor growth rate is an early indicator of antitumor drug activity in phase I clinical trials. Clin Cancer Res. 2014;20(1):246-52

12. Ferte $C$, Koscielny $S$, Albiges $L$, Rocher $L$, Soria JC, lacovelli $R$, Loriot $Y$, Fizazi K, Escudier B. Tumor growth rate provides useful information to evaluate sorafenib and everolimus treatment in metastatic renal cell carcinoma patients: an integrated analysis of the TARGET and RECORD phase 3 trial data. Eur Urol. 2014:65(4):713-20.

13. Ito T, Honma Y, Hijioka S, Kudo A, Fukutomi A, Nozaki A, Kimura Y, Motoi F, Isayama H, Komoto I, et al. Phase II study of lanreotide autogel in Japanese patients with unresectable or metastatic well-differentiated neuroendocrine tumors. Investig New Drugs. 2017;35(4):499-508.

14. Caplin ME, Pavel M, Cwikla JB, Phan AT, Raderer M, Sedlackova E, Cadiot G, Wolin EM, Capdevila J, Wall L, et al. Lanreotide in metastatic enteropancreatic neuroendocrine tumors. N Engl J Med. 2014;371(3):224-33.

15. Caplin ME, Pavel M, Cwikla JB, Phan AT, Raderer M, Sedlackova E, Cadiot G, Wolin EM, Capdevila J, Wall L, et al. Anti-tumour effects of lanreotide for pancreatic and intestinal neuroendocrine tumours: the CLARINET open-label extension study. Endocr Relat Cancer. 2016:23(3):191-9.

16. Gomez-Roca C, Koscielny S, Ribrag V, Dromain C, Marzouk I, Bidault F, Bahleda R, Ferte C, Massard C, Soria JC. Tumour growth rates and RECIST criteria in early drug development. Eur J Cancer. 2011;47(17):2512-6.

17. Stein WD, Gulley JL, Schlom J, Madan RA, Dahut W, Figg WD, Ning YM, Arlen PM, Price D, Bates SE, et al. Tumor regression and growth rates determined in five intramural $\mathrm{NCl}$ prostate cancer trials: the growth rate constant as an indicator of therapeutic efficacy. Clin Cancer Res. 2011;17(4): 907-17.

18. Stein WD, Wilkerson J, Kim ST, Huang X, Motzer RJ, Fojo AT, Bates SE. Analyzing the pivotal trial that compared sunitinib and IFN-alpha in renal cell carcinoma, using a method that assesses tumor regression and growth. Clin Cancer Res. 2012;18(8):2374-81.

19. Le Tourneau C, Servois V, Dieras V, Ollivier L, Tresca P, Paoletti X. Tumour growth kinetics assessment: added value to RECIST in cancer patients treated with molecularly targeted agents. Br J Cancer. 2012;106(5):854-7.

20. Liu Y, Litiere S, de Vries EG, Sargent D, Shankar L, Bogaerts J, Seymour L. The role of response evaluation criteria in solid tumour in anticancer treatment evaluation: results of a survey in the oncology community. Eur J Cancer. 2014;50(2):260-6.

21. Neal ML, Trister AD, Cloke T, Sodt R, Ahn S, Baldock AL, Bridge CA, Lai A, Cloughesy TF, Mrugala MM, et al. Discriminating survival outcomes in patients with glioblastoma using a simulation-based, patient-specific response metric. PLoS One. 2013;8(1):e51951.

22. Neal ML, Trister AD, Ahn S, Baldock A, Bridge CA, Guyman L, Lange J, Sodt $R$, Cloke $T$, Lai $A$, et al. Response classification based on a minimal model of glioblastoma growth is prognostic for clinical outcomes and distinguishes progression from pseudoprogression. Cancer Res. 2013;73(10):2976-86.

23. lacovelli R, Massari F, Albiges L, Loriot Y, Massard C, Fizazi K, Escudier B. Evidence and clinical relevance of tumor flare in patients who discontinue tyrosine kinase inhibitors for treatment of metastatic renal cell carcinoma. Eur Urol. 2015;68(1):154-60.

24. Barnes J, Johnson SJ, French JJ. Correlation of Ki-67 indices from biopsy and resection specimens of neuroendocrine tumours. Ann R Coll Surg Engl. 2017:99(3):193-7.
25. Kloppel G, La Rosa S. Ki67 labeling index: assessment and prognostic role in gastroenteropancreatic neuroendocrine neoplasms. Virchows Arch. 2018; 472(3):341-9.

26. Richards-Taylor S, Ewings SM, Jaynes E, Tilley C, Ellis SG, Armstrong T, Pearce $\mathrm{N}$, Cave J. The assessment of Ki-67 as a prognostic marker in neuroendocrine tumours: a systematic review and meta-analysis. J Clin Pathol. 2016;69(7):612-8.

27. Wolin EM, Caplin ME, Pavel ME, Ćwikła JB, Phan AT, Raderer M, Sedláčková E, Cadiot G, Capdevila J, Wall L, et al. Multivariate analysis of progression free survival in the CLARINET study of lanreotide autogel/depot vs placebo identifies prognostic factors in neuroendocrine tumors. Pancreas. 2016;45: 486.
Ready to submit your research? Choose BMC and benefit from:

- fast, convenient online submission

- thorough peer review by experienced researchers in your field

- rapid publication on acceptance

- support for research data, including large and complex data types

- gold Open Access which fosters wider collaboration and increased citations

- maximum visibility for your research: over $100 \mathrm{M}$ website views per year

At $\mathrm{BMC}$, research is always in progress.

Learn more biomedcentral.com/submissions 